\title{
Research on global energy system based on intelligent mobile Internet technology
}

\author{
Gang Wang ${ }^{1, a}$, Xingchuan Bao ${ }^{1, b}$, Hai Yu ${ }^{1, \mathrm{c}}$, Zhansheng Hou ${ }^{1, \mathrm{~d}}$, Liang Zhu ${ }^{1, \mathrm{e}}$, Haiyun Han ${ }^{1, \mathrm{f}}$, Lin Peng ${ }^{1, \mathrm{~g}}$ \\ ${ }^{1}$ State Grid Smart Grid Research Institute, Nanjing 211106, China \\ awanggang@sgri.sgcc.com.cn, bbaoxingchuan@sgri.sgcc.com.cn, 'yuhai@sgpi.sgcc.com.cn, 'houzhansheng@sgpi.sgcc.com.cn, \\ e zhuliang@sgpi.sgcc.com.cn, hanhaiyun@sgpi.sgcc.com.cn, ${ }^{\mathrm{g}}$ penglin @sgpi.sgcc.com.cn
}

\begin{abstract}
With the construction of the expansion of global energy system, the size of the grid is becoming larger and larger, the structure of maintenance person is becoming complex, communication and coordination are becoming more and more difficult. It is the main problem of operation and maintenance management on the energy Internet. It needs to study the global energy Internet system based on smart mobile Internet. This paper studies the global energy Internet operation and maintenance requirements, discusses the advantages and features of the intelligent mobile Internet technology, constructs the global energy network system of smart mobile Internet, which includes the layers of intelligent hardware, communication transmission, processing and others. In this paper, the key technologies of intelligent hardware, multi-source data fusion, image / speech recognition, scene computing, network and other key technologies are deeply studied. The global energy Internet system based on smart mobile Internet described in this paper can effectively solve the global energy Internet operation and maintenance difficulties, information access problem. With the progress of mobile Internet, artificial intelligence, wearable technology, the new smart mobile Internet operation and maintenance will continue to launch, and achieved a wide range of applications.
\end{abstract}

Keywords_global energy system; intelligent mobile Internet, smart mobile Internet.

\section{INTRODUCTION}

With the construction of the expansion of global energy system, the size of the grid is becoming larger and larger, the structure of maintenance person is becoming complex, communication and coordination are becoming more and more difficult. Equipment of the grid mainly includes all kinds of relay protection, the two system security, network equipment, protective equipment, wiring equipment and other equipment. It involves electric power information communication and other related professional. A large number of power grid equipment and the running time of different lengths are the main problem facing the energy Internet operation and maintenance management. With the advance of the construction of smart grid, the requirements for the reliability of the power grid is increasing. Future power grid will add node equipment as redundancy, resulting in a further increase in the number of network devices ${ }^{[1-2]}$. At the same time, with the improvement of the construction of "Three Grade Five" system, the further expansion of the personnel system. As well as in the field of engineers and other technical personnel to join the network operation and maintenance, making coordination work more difficult ${ }^{[3]}$. It must be updated to the current operation and maintenance system, to meet the requirements of operation and maintenance management in the energy internet. The mobile Internet technology is a hot spot of information energy infrastructure maintenance.

\section{AdVANTAGES AND FEATURES OF SMART MOBILE INTERNET TECHNOLOGY}

The development trend of intelligent mobile Internet has the following characteristics:1) The concept and scope of the mobile is expanding. It is no longer a simple concept of mobile terminal software. It is more and more embodied in the construction of hardware, network, process, application, data and infrastructure. Manufacturers and enterprises are gradually aware of this transformation, service and products will continue to expand. 2) The key technology is mature, the mobile communication network has gradually realized the integration, the broadband, the handset equipment intelligence, the multimedia, the mobile operating system, the development way diversification. 3) Application areas gradually are expanded, moving standards and norms continue to develop. Mobile security system continues to deepen. More enterprises implement the mobile strategy. Application domain has become greatly expanded. 4) Mobile industry chain gradually formed. 5) Business mobility shift from a single business to corporate level mobile strategy. Instant messaging, warehouse management, field operations, on-site sales service, customer oriented applications, on-site delivery and other business applications are facing new market opportunities, more and more applied to the enterprise.

Intelligent mobile Internet technology system includes intelligent hardware, multi-source data fusion, image / voice recognition, scene computing, network and other content, which can meet the global energy Internet operation and maintenance requirements, can achieve the polar, equatorial, desert and other areas of operation and maintenance requirements ${ }^{[4-5] .}$ Mobile Internet technology is currently being developed in the mobile terminal, access network, application services and network security 4 aspects of Technology. The technology of mobile terminal includes 3 kinds of terminal manufacturing technology, terminal hardware and software technology. Terminal manufacturing technology is a kind of integrated mechanical engineering, automation, information, electronic technology and other technology and equipment and 
system. Terminal hardware technology is the technology of realizing mobile information input, information output, information storage and processing. It is generally divided into the technology of processor chip, human-computer interaction, mobile terminal energy saving, mobile positioning technology. Processor chip technology is the core of the mobile Internet Computing and management control technology, responsible for receiving, storage, transmission, processing and power management, etc. Terminal software technology refers to the technology of data or information exchange between the user and the hardware interface and the mobile terminal, which is generally divided into mobile operating system, mobile middleware and mobile applications. Access network technology generally refers to two or more mobile terminal access to the Internet technology, including network access technology, mobile networking technology and network terminal management technology 3 categories. Mobile Internet access technology mainly includes: mobile communication network, wireless local area network (WLAN), wireless MESH network (WMN) access network technology, the integration of heterogeneous wireless network technology, etc. The technology of the mobile Internet is flexible and dynamic. Wireless local area network uses the star structure of the Ethernet, and the mobile communication network adopts centralized control and hierarchical structure. Both of them have central node. The mobile ad hoc network adopts the distributed networking technology, there is no central node, each node is a mutual relationship, can act as the role of the host and router. The wireless Mesh network uses a multi center self-organizing network technology, which is composed of a fixed Mesh router to use point to multi point method. The router manages the Mesh connection, the topology is relatively stable, and the transmission bandwidth is high. Mobile application service technology refers to the use of a variety of protocols or rules, to provide services to the mobile terminal technology, which is divided into front-end technology, backend technology and application layer network protocol 3 parts. Front end technology is used to show the content and logic implementation, including HTML, DOM, CSS, JavaScript and other technologies. Technology for the server side of the logic implementation and resource management, including database, dynamic web pages and other technologies. Application layer network protocol is used for information exchange and data transfer between the front and the back end, including HTTP, SMTP, FTP protocol, etc. The key technologies of the application are HTML5, mobile search, mobile social networks, Web real-time communication. Mobile network security technology is mainly divided into mobile terminal security, mobile security, mobile application security and location privacy protection technology. Mobile terminal security mainly includes terminal equipment safety and the information content security, such as information content is illegal tampering and access, or modify the useful information of the terminal through the operating system, using viruses and malicious code of system and destruction, may also be unauthorized access to a variety of Internet resources, the leakage of private information etc. It mainly includes the user information encryption storage technology, software signature technology, virus (Mu Ma) protection technology, the host firewall technology, etc.

\section{GLOBAL ENERGY GRID SYSTEM FOR SMART MOBILE INTERNET}

Intelligent hardware includes wearable, intelligent terminal, robot, unmanned aerial vehicle and other technologies, which can achieve global energy network operation and maintenance information collection.

Communication transmission includes long-distance wireless communication, optical wireless communication, heterogeneous network access, SDN network technology, and so on, which can achieve the global energy network operation and maintenance information reliable transmission.

Interactive technology includes multi-source data fusion, image / speech recognition, scene computing and other technologies to achieve global energy network operation and maintenance information real-time processing. After intelligent, hardware has the ability to connect. Hardware and hardware are connected to each other, the hardware and the mobile phone are connected, and the hardware and the people also realize the connection. After the connection, the hardware implementation of the load of the Internet service. It forms a "cloud + end" of the typical structure, with the added value of big data. Intelligent hardware is characterized in that the function can be expanded, product fast iteration. It can automatically sense, self study, realize the device, between people, and between the environment, and the Internet is closely linked. It is equipped with embedded computing platform for image transmission, identification and analysis of judgment conductor temperature, rivets elastic, insulator contamination degree, tower inclination, judgment of transformer oil temperature, equipment damage, the meter reading and other.

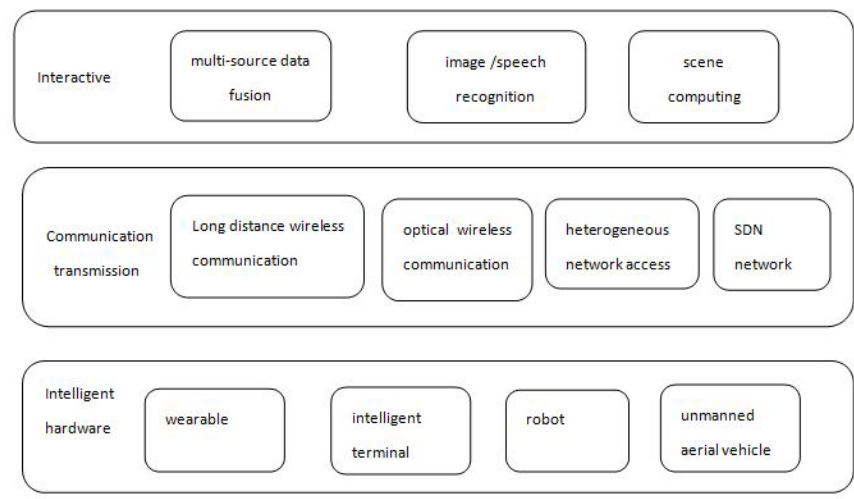

Fig. 1. Intelligent mobile Internet technology system of global energy network

\section{KEY TECHNOLOGY}

\section{A. Intelligent hardware}

Intelligent hardware adopt the combination of hardware and software to transform the traditional device and then let it have a smart feature. Transformed objects may be electronic devices, such as watches, televisions and other electrical appliances. It may also have no electronic devices, such as door locks, cups, cars and even houses. 
Intelligent hardware is an important front medium of intelligent mobile internet. Intelligent hardware technology is based on the following four aspects.1) Internet of things: The technology of sensor, communication, terminal computing, and the Internet of things have laid the foundation for the automatic induction of the intelligent hardware and the connection of each other. 2) Cloud computing: The local computing power of intelligent hardware is limited, which needs to store data in the cloud. The cloud also need to share more computing, such as LBS, voice recognition, big data. 3)Intelligent terminal: IPhone and Android set off a wave of mobile phones for intelligent hardware to prepare a large number of developers, but also to develop the market. Intelligent mobile phone has become a fact of personal computing, data and control center, which is the interface between the intelligent hardware and human. 4) Wireless communication: The popularity of WIFI, the popularity of mobile cellular networks, the popularity of Bluetooth 4 and the technology of address fence, and the popularity of 4G, provide a basis for information exchange between intelligent hardware.

Intelligent hardware includes various types of wearable devices for different tasks, such as helmets, glasses, bracelets, foot ring, etc. It integrates different sensors, to provide convenient information collection, access and interaction of the terminal, such as the smart Helmet Camera collection site information, bracelet monitoring personnel signs, environmental information, foot ring precision measurement position information, etc. Maintenance person adopts the perception of wearable devices for the scene, to complete the navigation route planning, spare parts preparation, coordination of field operations, the realization of safety inspection. Maintenance problems such as the emergence of the problem can not be solved independently, which can directly connect to the background support departments, realtime interactive.

Unmanned airborne equipment and robot equipment in the intelligent equipment are used in a more diverse operating scenarios. The unmanned aerial vehicles or robots collect various types of human information difficult to gather by human, rich power mobile Internet mobile device type. The UAV is equipped with visible light, infrared camera to capture the image information, line tower. Inspection personnel directly view tower, lines and other equipment with the UAV angle. Robots, unmanned aerial vehicles are equipped with a variety of sensing devices and environmental information, such as automatic acquisition of equipment and environmental information.

\section{B. Multi-source data fusion}

The data fusion technology will be processed from multiple sensors or multi-source information, and the results are more accurate and reliable. More stringent definition: the use of computer technology to the timing of a number of sensor observation information in a certain criteria to be automatically analyzed and integrated in order to complete the process of information processing required for decision- making and estimation tasks ${ }^{[6]}$. The basic principle of multi sensor information fusion technology is also full use of multiple sensor resources, as well as the human brain processing information. Through the reasonable control and use of these sensors and their observation information, it combines the redundant or complementary information in time and space with some criteria to obtain the consistency of the observed object. The basic objective of data fusion is to export more effective information through the combination of data optimization. Its ultimate goal is to improve the effectiveness of multiple sensor systems by using multiple sensors to joint or combined operations. Data fusion is mainly divided into two categories: The first type is local or self - owned. It collects data from multiple sensors on a single platform. The second category is global or regional integration. It optimizes the combination of multiple platforms, multiple sensor data from space and time.

According to the 3 levels of data abstraction, fusion can be divided into 3 categories: data level fusion, feature level fusion and decision level fusion. Data fusion is a kind of data synthesis and processing technology. It is the integration and application of many traditional subjects and new technologies, such as communication, pattern recognition, decision theory, uncertainty theory, signal processing, estimation theory, optimization technology, computer science, artificial intelligence and neural network. In recent years, many scholars have introduced genetic algorithm, wavelet analysis technology, virtual technology into data fusion.

\section{Image / speech recognition}

With the mobile Internet into the fast lane, mobile terminal users of mobile data business needs is increasingly strong, the user wants to make full use of the current equipment to provide more value-added services ${ }^{[7]}$. The number of images on the current mobile Internet is growing rapidly, although a large number of users are attracted by the rich image information in the mobile Internet, but it is difficult to find the real needs of the information in mass data environment. Mobile Internet has an urgent need for image recognition technology, needs to have accurate and efficient image recognition tools, makes the real life and virtual world more closely linked together to get a better mobile Internet experience.

The research object of image recognition technology is based on the observed images, and the objects of the image are classified into the category and make a meaningful judgment. It uses modern information processing and computing technology to simulate and complete the process of human knowledge. Generally speaking, an image recognition system mainly consists of three parts, namely, image segmentation, image feature extraction and classification of classifier.

Image segmentation divides the image into several meaningful regions. Then the image is extracted and the final classifier is classified according to the extracted image features. In fact, image recognition and image segmentation do not exist in strict boundaries. In a sense, the process of image segmentation is the process of image recognition. Image segmentation focuses on the relationship between 
object and background, the study of the overall attributes of objects in a specific context, while the image recognition is focused on the properties of the object itself.

\section{Scenario calculation}

For the future of the Internet and intelligent power grid terminal access, a wide range of information applications should be open and interactive, analysis and decision-making should be a high degree of intelligence. Supply side and demand side need to achieve two-way interaction and diversified service requirements. It needs to realize the intelligent interaction between the user and the energy Internet, highly efficient and interactive, and assist the user to make the best decision, and provide users with professional, personalized, customized services.

(1) situational information perception and recognition technology: research based on the position, behavior, the task and other information technology;

(2) the evolution mechanism of the situation fusion: the research on the control strategy, the feature base and the fusion algorithm of the intelligent mobile internet;

(3) the construction of information space: the research on information model framework and application technology of information model; the research on the process of power grid equipment perception, data processing, decision analysis and data processing strategy.

(4) scenario reasoning and decision making mechanism: Based on scenario driven reasoning engine, the research of the iterative and evolutionary mechanism of scenario rules, the coordination and application of scene information.

(5) the scene knowledge base construction: it constructs the scene ontology library, the rule base, the service ontology, the target storage library.

\section{E. Network}

SDN is based on the OpenFlow network protocol, which is designed and made by Stanford University in order to construct the network innovation platform. The core idea of SDN is to enhance the ability of the network through the standardization of the hardware, the network level of the control and forwarding and separation of the device ${ }^{[8]}$.A SDN network contains 3 levels of Architecture: the underlying physical network (such as switches, etc.), SDN controller, SDN application. In the typical SDN architecture definition, the application layer is the top layer, including a variety of business and Application. The control layer is responsible for processing the layout of the data plane resources, maintaining the network topology and state information. The infrastructure layer is responsible for data processing, forwarding and state collection based on flow table. The SDN network uses the architecture of control plane and data plane to separate all the advanced processing functions (such as network management control and load balance), which is used to transmit the advanced processing functions. The controller may be a proprietary hardware device, or a virtual machine, or a physical server. It is similar to the computer "central processor" role, mainly for the exchange of data forwarding for the best design and provide more application services. Communication between the controller and the switch adopt the OpenFlow network protocol, the SDN controller is responsible for sending out the control instruction, the switch is responsible for receiving the instruction and carries on the corresponding data forwarding. The controller is in fact only a "platform", and it is the role of the network application to provide a unified operating environment. The advanced processing functions of the controller are the "application" (also is the SDN application). The user can develop a variety of network applications, and define the business processing logic of the network through software, and realize the efficient and flexible network management and control. For example, the ability to program is conducive to the network innovation, centralized control and simplify the network management, the global network view to make the network fine-grained control possible. Virtual support network resource optimization and scheduling and efficient use of the Internet model is being deformed into a fast changing between the operators, in order to the contents of the center of the relationship, rather than in the past that level of the slowly changing access mode. Users are free to develop custom switch (Network) functions, and do not need to be subject to network equipment manufacturers.

\section{CONCLUSIONS}

With rapid development of global energy Internet, based on Intelligent Mobile Internet operation and maintenance technology will play an increasingly important role. With the progress of mobile Internet, artificial intelligence, wearable technology, the new smart mobile Internet operation and maintenance will continue to launch, and achieved a wide range of applications.

\section{References}

[1] RIFKIN J.The third industrial revolution:how lateral power is transforming energy,the economy, and the world [M].New York:Palgrave MacMillan,2011.

[2] HUANG A Q,CROW M L,HEYDT G T,et al.The future renewable electric energy delivery and management (FREEDM) system:the energy internet[J].Proceedings of the IEEE,2011, 99(1):133-148.

[3] ZHANG Z, CHOW M Y.Convergence analysis of the incremental cost consensus algorithm under different communication network topologies in a smart grid[J].IEEE Trans on Power Systems,2012,27(4):1761-1768.

[4] ALMASSALKHI M,HISKENS I.Optimization framework for the analysis of large-scale networks of energy hubs[C]//Proceedings of 17th Power System Computation Conference(PSCC),August 2226,2011,Stockholm,Sweden:7p.

[5] ZHAO Junhua,WEN Fushuan,XUE Yusheng,et al.Cyber physical power systems: architecture, implementation techniques an

[6] ZHAO Junhua, WEN Fushuan, XUE Yusheng, et al.Modeling analysis and control research framework of cyber physical power systems [J ].Automation of Electric Power Systems,2011,35(16):1-8.

[7] Cao Junwei, Yang Mingbo, Zhang Dehua, et al. Energy internet : an infrastructure for cyber-energy integration[J] . Southern Power System Technology, 2014, 8(4) : 1-10.

[8] Cao Junwei, Meng Kun, Wang Jiye, et al. Energy internet and energy router[J] . Chinese Science: Information Science, 2014, 44(6) : 714-727. 